\title{
Tumor Oxygenation
}

National Cancer Institute

\section{Source}

National Cancer Institute. Tumor Oxygenation. NCI Thesaurus. Code C19557.

Level of oxygen saturation within a tumor. Increase in the oxygenation of a tumor acts as a radiosensitizer. 